\title{
Dynamical particles in spatially flat FLRW space-times
}

\author{
Ion I. Cotăescu ${ }^{\mathrm{a}}(1)$ \\ West University of Timişoara, V. Pârvan Ave. 4, 300223 Timişoara, Romania
}

Received: 4 October 2021 / Accepted: 12 January 2022 / Published online: 28 January 2022

(C) The Author(s) 2022

\begin{abstract}
Metrics of dynamical point particles embedded in spatially flat FLRW space-times are derived as isotropic solutions of the Einstein equations with the energymomentum tensor of a perfect fluid. These particles are produced by central singularities of the fluid density but without changing the pressure of the asymptotic FLRW spacetimes. It is shown that each particle gets a dynamical photon sphere before a critical instant when two dynamical apparent horizons arise just on the photon sphere evolving then as $\mathrm{C}$-curves. The angular radius of the shadow associated to the photon sphere is derived and compared with the known results.
\end{abstract}

\section{Introduction}

The static non rotating black holes with spherical symmetry are solutions of the Einstein or Einstein-Maxwell equations without other gravitational sources apart from the optional cosmological constant $\Lambda$. These equations were solved in static local charts (called here frames) either analytically, finding the classical black hole solutions [1], or sequentially as in the Bondi-Sachs approach [2-4].

On the other hand, in cosmology one considers evolving Friedmann-Lemaître-Robertson-Walker (FLRW) spacetimes which are plausible models of our universe in various epochs of evolution. Each FLRW space-time evolves according to its scale factor $a(t)$ which solves the Einstein equations with the energy-momentum tensor of an isotropic perfect fluid. As it is somewhat paradoxical to populate such backgrounds with static black holes, one looks for dynamical point particles that could be embedded in the perfect fluid of any FLRW space-time. An example is the McVittie class of exact solutions [5] of the Einstein equations with perfect fluid having FLRW asymptotic limits and the Schwarzschild limit when $a(t)=1$. These metrics describing dynamical

a e-mail: i.cotaescu@e-uvt.ro (corresponding author) point particles were studied mainly in physical frames with Painlevé-Gullstrand coordinates [6,7] where their properties are visible and intuitive [8-10] and the generalization to FLRW space-time of any space curvature was possible [11].

The McVittie particle modifies the pressure of the fluid adding a singularity on the Schwarzschild sphere but without changing the density. We may ask if a complementary solution, changing the density but preserving the pressure, could be found in the frames with physical coordinates. Trying to fix this problem we devote the present letter to a new class of metrics in physical frames which seem to describe dynamical point particles carried by the isotropic perfect fluids of the FLRW space-times. These metrics are exact solutions of the Einstein equations with a perfect fluid with the same pressure as in the FLRW space-times but giving rise to a point singularity in its density. Our main goal is to investigate the specific properties of these metrics which could reveal black hole features.

We start in the next section inspecting briefly the static black holes in physical frames focusing the on the McVittie metrics describing dynamical particles. Section 3 is devoted to our new isotropic solution for which we derive the term added to the fluid density for satisfying the Einstein equations. Once we have the solutions in physical coordinates we introduce the co-moving space coordinates and a new radial coordinate that puts the metric in diagonal form. Hereby we identify the areal radius which will be used in the next section for finding the apparent horizons. We show that these dynamical particles do not have horizons until a critical instant when a pair of dynamical horizons are arising simultaneously on the same sphere. When the time is increasing these evolve as fold-type (or C-curves) horizons, one expanding as a cosmological horizon, approaching asymptotically to the apparent horizon of the asymptotic FLRW space-time, while the second one is shrinking as a black hole horizon. We meet thus the same phenomenology as in the case of the McVittie dynamical particles $[8,12]$. In Sect. 4 we derive the radial null geodesics showing that their complicated equation may 
be integrated sequentially by using an algorithm of perturbations. The light around the dynamical particles is studied in Sect. 5 where we deduce the radius of the dynamical photon sphere which appears before the apparent horizons shoving that at the critical instant the horizons arise just on this sphere. A possible dynamical shadow measured by a remote observer is discussed and compared with previous results. In the last section we present our concluding remarks.

We use the Planck units with $\hbar=c=G=1$.

\section{Black holes in physical frames}

The principal black holes solutions with spherical symmetry are written in static frames, $\left\{t_{s}, \mathbf{x}\right\}$, with coordinates $x^{\mu}$ $(\alpha, \mu, v, \ldots=0,1,2,3)$, i. e. the static time $x_{s}^{0}=t_{s}$ and Cartesian space coordinates $\mathbf{x}=\left(x^{1}, x^{2}, x^{3}\right)$ associated to the spherical ones $(r, \theta, \phi)$. The geometry of these black holes is defined by line elements of the form

$\mathrm{d} s^{2}=f(r) \mathrm{d} t_{s}^{2}-\frac{\mathrm{d} r^{2}}{f(r)}-r^{2} \mathrm{~d} \Omega^{2}$,

where $\mathrm{d} \Omega^{2}=\mathrm{d} \theta^{2}+\sin ^{2} \theta \mathrm{d} \phi^{2}$. The function $f$ determines the properties of the black hole and the asymptotic form of the space-time. For example, in the case of a charged ReissnerNordstrom-de Sitter black hole, the function

$f(r)=1-\frac{2 m}{r}+\frac{q^{2}}{r^{2}}-\omega_{d S}^{2} r^{2}, \quad \omega_{d S}=\sqrt{\frac{\Lambda}{3}}$,

depends on the black hole mass $m$ and charge $q$ while the Hubble-de Sitter constant $\omega_{d S}$ gives the asymptotic behaviour of the background. Thus we may say that when $\omega_{d S} \neq 0$ the black hole stays in a de Sitter universe while for $\omega_{d S}=0$ it stays in the Minkowski space-time. However, physically speaking, the de Sitter manifold is not a static space-time as its expanding portion is a model of expanding universe governed by the cosmic, or proper, time $x^{0}=t$ defined as

$t=t_{s}-\int \mathrm{d} r \frac{\sqrt{1-f(r)}}{f(r)}$.

Substituting $t_{s} \rightarrow t$ we obtain the frame $\{t, \mathbf{x}\}$, with physical coordinates of Painlevé-Gullstrand type [6,7], in which the static metric (1) takes the form

$\mathrm{d} s^{2}=f(r) \mathrm{d} t^{2}+2 \sqrt{1-f(r)} \mathrm{d} t \mathrm{~d} r-\mathrm{d} r^{2}-r^{2} \mathrm{~d} \Omega^{2}$,

laying out flat space sections. In this manner, one may introduce physical frames in any isotropic manifold with flat space sections.

We have thus the opportunity of studying the dynamical black holes with spherical symmetry in their proper physical frames with line elements of the general form

$$
\mathrm{d} s^{2}=g_{00}(t, r) \mathrm{d} t^{2}+2 g_{0 r}(t, r) \mathrm{d} r \mathrm{~d} t
$$

$$
-g_{r r}(r) \mathrm{d} r^{2}-r^{2} \mathrm{~d} \Omega^{2} .
$$

This metric encapsulates the black hole properties and the geometry of the asymptotic space-time which hosts the black hole. The black hole is imagined then as a point dynamical particle whose metric tensor is an exact solution of the Einstein equations

$G_{v}^{\mu}=\Lambda \delta_{v}^{\mu}+8 \pi\left[(\rho+p) U^{\mu} U_{v}-p \delta_{v}^{\mu}\right]$,

in a perfect fluid of density $\rho$ (of matter or energy) and pressure $p$, moving with the four-velocity $U^{\mu}$ with respect to the physical frame under consideration. In a proper co-moving frame where the four-velocity has the components

$U_{\mu}=\left(\frac{1}{\sqrt{g^{00}}}, 0,0,0\right), \quad U^{\mu}=g^{\mu 0} U_{0}=\frac{g^{\mu 0}}{\sqrt{g^{00}}}$,

Eq. (6) is solved by an isotropic Einstein tensor whose nonvanishing components satisfy

$$
\begin{aligned}
& G_{r}^{r}=G_{\theta}^{\theta}=G_{\phi}^{\phi} \equiv G, \\
& G_{0}^{r}=\frac{g^{0 r}}{g^{00}}\left(G_{0}^{0}-G\right) .
\end{aligned}
$$

Moreover, the asymptotic manifold must also be a solution of the Eq. (6) corresponding to the asymptotic gravitational sources.

The asymptotic space-times considered so far are either flat ones or spatially flat FLRW space-times with scale factors $a(t)$ we denote here by $(M, a)$ understanding that $(M, 1)$ is just the Minkowski space-time. Thus, for $r \rightarrow \infty$, the line element (5) is supposed to take the asymptotic form

$\mathrm{d} s^{2}=\left(1-\frac{\dot{a}^{2}}{a^{2}} r^{2}\right) \mathrm{d} t^{2}+2 \frac{\dot{a}}{a} r \mathrm{~d} r \mathrm{~d} t-\mathrm{d} r^{2}-r^{2} \mathrm{~d} \Omega^{2}$,

in the physical frames $\{t, \mathbf{x}\}$ of the space-times $(M, a)$. This line element depends only on the Hubble function $\frac{\dot{a}(t)}{a(t)}$ which gives the radius

$r_{a}(t)=\left|\frac{a(t)}{\dot{a}(t)}\right|$,

of the dynamical apparent horizon. The Einstein tensors of these manifolds have diagonal elements giving the Friedmann equations

$$
\begin{aligned}
G_{0}^{0}(a) & =3 \frac{\dot{a}^{2}}{a^{2}}=\Lambda+8 \pi \rho_{a}, \\
G(a) & =2 \frac{\ddot{a}}{a}+\frac{\dot{a}^{2}}{a^{2}}=\Lambda-8 \pi p_{a},
\end{aligned}
$$

and a non-diagonal one, $G_{0}^{r}(a)$, satisfying the condition (9). The gravitational sources of the asymptotic space-time $(M, a)$ are the asymptotic density and pressure, $\rho_{a}=$ $\lim _{r \rightarrow \infty} \rho$ and $p_{a}=\lim _{r \rightarrow \infty} p$.

In this framework many models of dynamical particles are considered so far (see for instance [13]) among them 
a prominent approach is the McVittie [5] class of metrics describing isotropic dynamical particles in any spatially flat FLRW. In physical frames, the McVittie line element is given by Eq. (5) where the components of the metric tensor depend only on the black hole mass $m$ and Hubble's function as [11]

$$
\begin{aligned}
& g_{00}(t, r)=1-\frac{2 m}{r}-\frac{\dot{a}(t)^{2}}{a(t)^{2}} r^{2}, \\
& g_{0 r}(t, r)=\frac{\dot{a}(t)}{a(t)} \frac{r}{\sqrt{1-\frac{2 m}{r}}}, \quad g_{r r}(r)=\frac{1}{1-\frac{2 m}{r}} .
\end{aligned}
$$

In the asymptotic limit, when $r \rightarrow \infty$, this line element takes the form (10) as in the physical frame of the spacetime $(M, a)$. However, in the flat limit, when $a(t) \rightarrow 1$ and $\dot{a}(t) \rightarrow 0$, this becomes the Schwarzschild line element of the static frame (1) with $f=1-\frac{2 m}{r}$ instead of the physical one (4). The metric tensor defined by Eqs. (14) and (15) is an exact solution of Eq. (6) with [11]

$$
\begin{aligned}
& 8 \pi \rho=G_{0}^{0}=G_{0}^{0}(a), \\
& 8 \pi p=-G=-G_{0}^{0}(a)-\frac{1}{\sqrt{1-\frac{2 m}{r}}}\left[G(a)-G_{0}^{0}(a)\right],
\end{aligned}
$$

while the component $G_{0}^{r}$ satisfies the condition (9). Thus the presence of this dynamical particle modifies only the pressure of the fluid, introducing a Schwarzschild singularity, but without affecting the density. It is remarkable that there is a critical instant when a pair of dynamical horizons appears, one expanding asymptotically to the apparent horizon of the asymptotic FLRW space-time while the second one is shrinking to the Schwarzschild sphere of radius $2 m[8,12]$. Note that a similar behaviour was observed recently $[13,14]$ in the Lemaître-Tolman-Bondi (LTB) space-times [15].

\section{Isotropic dynamical particles}

Under such circumstances, we may ask if other solutions of the Einstein equations could be found as singularities of the density of the perfect fluid but without changing its pressure. In what follows we try to find an answer looking for new exact solutions of the Einstein equations in space-times with perfect fluid assuming that these must have: line elements (5) with flat space sections (i. e. $g_{r r}=1$ ), the asymptotic form (10) and flat limit (4). For investigating this problem we consider a new class of metrics in which the mass term $\frac{2 m}{r}$ is introduced independently, without using the traditional Schwarzschild's binomial $1-\frac{2 m}{r}$. These are supposed to have the line elements of the form

$$
\begin{aligned}
\mathrm{d} s^{2}= & \mathrm{d} t^{2}-\left[\mathrm{d} r-h_{s}(t, r) \mathrm{d} t\right]^{2}-r^{2} \mathrm{~d} \Omega^{2} \\
= & {\left[1-h_{s}(t, r)^{2}\right] \mathrm{d} t^{2}+2 h_{s}(t, r) \mathrm{d} r \mathrm{~d} t } \\
& -\mathrm{d} r^{2}-r^{2} \mathrm{~d} \Omega^{2},
\end{aligned}
$$

where

$h_{s}(t, r)=\frac{\dot{a}(t)}{a(t)} r+\sqrt{\frac{2 M_{s}(t)}{r}}, \quad M_{s}(t)=\frac{m}{a(t)^{s}}$.

In this metric, $M_{s}(t)$, with $s \in \mathbb{R}$, is the dynamical mass depending on the invariant mass $m$ and the scale factor.

The Einstein tensors of these manifolds have the nonvanishing diagonal components

$$
\begin{aligned}
G_{0}^{0} & =G_{0}^{0}(a)+\delta_{s}, \\
G_{r}^{r} & =G(a)+\left(1-\frac{s}{3}\right) \delta_{s}, \\
G_{\theta}^{\theta}=G_{\phi}^{\phi} & =G(a)+\frac{1}{4}\left(1-\frac{s}{3}\right) \delta_{s},
\end{aligned}
$$

where

$\delta_{s}(t, r)=3 \frac{\dot{a}(t)}{a(t)} \sqrt{\frac{2 M_{s}(t)}{r^{3}}}$,

while the non-diagonal one accomplishes the condition

$G_{0}^{r}=\frac{g^{0 r}}{g^{00}}\left(G_{0}^{0}-G_{r}^{r}\right)$.

Hereby we understand that for $s=3$ we obtain the desired solution of Eq. (6) with isotropic Einstein tensor which satisfies both the conditions (8) and (9). Note that the metrics with $s \neq 3$ may also be seen as solutions of the Einstein equations but with anisotropic gravitational sources given by supplemental terms added to the energy-momentum tensor of the perfect fluid.

Restricting ourselves to the isotropic solutions with $s=3$ we denote by $(M, a, m)$ the space-times with the line elements in physical frames,

$\mathrm{d} s^{2}=\mathrm{d} t^{2}-[\mathrm{d} r-h(t, r) \mathrm{d} t]^{2}-r^{2} \mathrm{~d} \Omega^{2}$,

where now

$h(t, r)=h_{s=3}(t, r)=\frac{\dot{a}(t)}{a(t)} r+\sqrt{\frac{2 M(t)}{r}}$,

depends on the dynamical mass,

$M(t)=M_{s=3}(t)=\frac{m}{a(t)^{3}}$.

The corresponding Einstein tensors have isotropic components,

$$
\begin{aligned}
G_{0}^{0} & =G_{0}^{0}(a)+\delta, \\
G & =G(a),
\end{aligned}
$$

where $G_{0}^{0}(a)$ and $G(a)$ are the components the Einstein tensor of the asymptotic manifold $(M, a, 0) \equiv(M, a)$ as given 
by Eqs. (12) and (13). These solutions describe dynamical particles whose presence gives rise to the additional term

$\delta(t, r)=\delta_{s=3}(t, r)=3 \frac{\dot{a}(t)}{a(t)} \sqrt{\frac{2 M(t)}{r^{3}}}$,

which modifies the density of the perfect fluid, $8 \pi \rho_{a} \rightarrow 8 \pi \rho=$ $8 \pi \rho_{a}+\delta$, introducing a central singularity in $r=0$, while the fluid pressure remains unchanged, $p=p_{a}$. For this reason we believe that these isotropic solutions can be interpreted as dynamical point particles carried by the perfect fluids which govern the evolution of their backgrounds.

For studying these particles we need to introduce other types of coordinates in the space-times $(M, a, m)$. We start with the FLRW ones, $\{t, \hat{\mathbf{x}}\}$, formed by the cosmic time and the co-moving space coordinates $\hat{\mathbf{x}}$ related to the physical ones as $\mathbf{x}=\hat{\mathbf{x}} a(t)$. The corresponding frame with spherical coordinates, $\{t, \hat{r}, \theta, \phi\}$, has the line element

$$
\begin{aligned}
\mathrm{d} s^{2}= & \mathrm{d} t^{2}-a(t)^{2} \\
& \times\left[\frac{1}{\hat{r}}\left(\sqrt{\hat{r}} \mathrm{~d} \hat{r}-\sqrt{2 m} \frac{\mathrm{d} t}{a(t)^{3}}\right)^{2}+\hat{r}^{2} \mathrm{~d} \Omega^{2}\right]
\end{aligned}
$$

obtained after substituting $r=\hat{r} a(t)$ in Eq. (25). In the round parentheses we separated the exact 1 -form $\mathrm{d} \bar{r}$ giving the new radial coordinate

$\bar{r}=\frac{2}{3} \hat{r}^{\frac{3}{2}}-\sqrt{2 m} \int_{t_{0}}^{t} \frac{\mathrm{d} t^{\prime}}{a\left(t^{\prime}\right)^{3}}$,

of the frame $\{t, \bar{r}, \theta, \phi\}$ which has the diagonal line element

$\mathrm{d} s^{2}=\mathrm{d} t^{2}-a(t)^{2}\left[\frac{\mathrm{d} \bar{r}^{2}}{\hat{r}(t, \bar{r})}+\hat{r}(t, \bar{r})^{2} \mathrm{~d} \Omega^{2}\right]$,

where the new function

$\hat{r}(t, \bar{r})=\left(\frac{3}{2}\right)^{\frac{2}{3}}\left(\bar{r}+\sqrt{2 m} \int_{t_{0}}^{t} \frac{\mathrm{d} t^{\prime}}{a\left(t^{\prime}\right)^{3}}\right)^{\frac{2}{3}}$,

results from Eq. (32). The time $t_{0}$ gives the arbitrary instant when $\hat{r}$ and $\bar{r}$ may vanish simultaneously, $\bar{r}=0 \rightarrow$ $\hat{r}\left(t_{0}, 0\right)=0$. Note that the metric (33) is close to the LTB metrics [15] apart from the time dependence which here is of FLRW type. In fact the metric (33) looks like a hybrid of these two types of mentioned metrics.

\section{Horizons}

The additional term (30) vanishes in the flat limit $\left(\frac{\dot{a}}{a} \rightarrow 0\right)$ when the metric (25) becomes just the Schwarzschild one in the physical frame. This suggests us that the new dynamical particles studied here may behave as black holes at least on some time domains of the expanding space-times with positive Hubble functions and $h(t, r)>0$. For investigating this possibility we start looking for the apparent horizons that could play the role of dynamical black hole or cosmological horizons.

These horizons can be found as the solutions of the equation $\nabla^{\mu} R \nabla_{\mu} R=0$ satisfied by the areal radius $R[16,17]$ which in our case can be identified in the line element (33) as $R(t, \bar{r})=a(t) \hat{r}(t, \bar{r})$. Then after a few manipulation we find that the equation

$\nabla^{\mu} R \nabla_{\mu} R=\left[\partial_{t} R(t, \bar{r})\right]^{2}-\frac{\hat{r}(t, \bar{r})}{a(t)^{2}}\left[\partial_{\bar{r}} R(t, \bar{r})\right]^{2}=0$

is (up to sign) just the equation $g_{00}(t, r)=1-h(t, r)^{2}=0$ in the physical frame. This can be spit in two equations $h(t, r)=$ \pm 1 which do not hold simultaneously. As mentioned before, in what follows we restrict ourselves to the expanding spacetimes with $h(t, r)>0$ keeping the equation $h(t, r)=1$ only. Observing that this is not an algebraic equation in $r$, we introduce the new variable $x=\sqrt{r}>0$ and we solve the equation $h\left(t, x^{2}\right)=1$ obtaining the solutions listed in the Appendix.

These solutions get a physical meaning at the critical time $t_{c r}$ when $\Delta\left(t_{c r}\right)=0$. For $t<t_{c r}$ there are no solutions with physical meaning. Whether $t>t_{c r}$ then we have two positive solutions giving the horizons of radii (A.3) and (A.2). For understanding their role we resort to the expansions

$$
\begin{aligned}
r_{b}(t)= & 2 M(t)\left[1+\frac{4 M(t)}{r_{a}(t)}+\mathscr{O}\left(\frac{M(t)^{2}}{r_{a}(t)^{2}}\right)\right], \\
r_{c}(t)= & r_{a}(t)\left[1-\sqrt{\frac{2 M(t)}{r_{a}(t)}}-\frac{M(t)}{r_{a}(t)}\right. \\
& \left.-\frac{5}{2 \sqrt{2}} \frac{M(t)^{\frac{3}{2}}}{r_{a}(t)^{\frac{3}{2}}}+\mathscr{O}\left(\frac{M(t)^{2}}{r_{a}(t)^{2}}\right)\right],
\end{aligned}
$$

in a time domain where $M(t) \ll r_{a}(t)$. Hereby we see that there are two dynamical horizons, a black hole one of radius $r_{b}(t)$ and a cosmological one on the sphere of radius $r_{c}(t)$. The evolution of these horizons and their relative positions with respect to the asymptotic horizons of radii $r_{a}(t)$ may be illustrated analysing two simple examples.

Example 1 Let us consider first a dynamical particle in the space-time $\left(M, t^{\frac{2}{3}}, m\right)$ with the scale factor $a(t)=t^{\frac{2}{3}}$ whose asymptotic background $\left(M, t^{\frac{2}{3}}\right)$ is just the matter-dominated universe. The gravitational sources producing this dynamical particle,

$8 \pi \rho=G_{0}^{0}(a)+\delta=\frac{4}{3 t^{2}}\left(1+\frac{3}{2} \sqrt{\frac{2 m}{r^{3}}}\right)$,

$8 \pi p=G(a)=0$,

preserve the null pressure while the density kips the big bang singularity in $t=0$ which means that the condition $t>0$ remains mandatory. In this geometry we can solve the equation $\Delta\left(t_{c r}\right)=0$ finding the critical time $t_{c r}=(9 m)^{\frac{1}{3}}$. The 

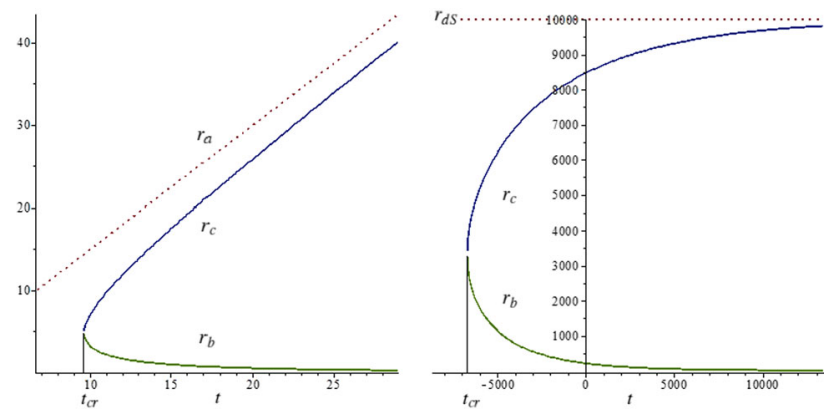

Fig. 1 Fold-type, or C-curve, evolution of the dynamical horizons arising at the critical time $t_{c r}$ in the matter-dominated universe (left panel) and de Sitter expanding universe (right panel). The cosmological horizons tend to the apparent horizons of the asymptotic FLRW space-times while the black hole horizons collapse

dynamical particle does not have horizons for $t<t_{c r}$ but when $t>t_{c r}$ the cosmological and black hole dynamical horizons evolves as in the left panel of Fig. 1. The cosmological horizon of radius $r_{c}(t)$ approaches asymptotically to the apparent horizon of $(M, a)$ which is a sphere of radius $r_{a}(t)=\frac{3}{2} t$ expanding with the constant velocity $v_{a}=\frac{3}{2}$. The black hole horizon is shrinking to the dynamical sphere of radius $2 M(t)=2 m t^{-2}$.

Example 2 The second example is of a dynamical particle in space-times $\left(M, a_{d S}, m\right)$ having the de Sitter scale factor, $a_{d S}(t)=\exp \left(\omega_{d S} t\right)$ with $\omega_{d S}$ as in Eq. (2), defined for $t \in \mathbb{R}$. Consequently, the asymptotic space-time $\left(M, a_{d S}\right)$ is just the de Sitter one whose static horizon on the sphere of radius $r_{a}=\omega_{d S}^{-1}$ is an event horizon separating two different domains of causality $[18,19]$. Moreover, this manifold can host its own Schwarzschild-de Sitter black hole which is a vacuum solution of the Einstein equations with cosmological constant and Einstein tensor $G_{\mu}^{v}\left(a_{d S}\right)=\delta_{\mu}^{v} \Lambda$. The dynamical particle in the space-time $\left(M, a_{d S}, m\right)$ is different as this is a perturbation of a perfect fluid filling out the former empty de Sitter space-time. This modifies the Einstein tensor which now reads

$$
\begin{aligned}
G_{0}^{0} & =G_{0}^{0}\left(a_{d S}\right)+\delta=\Lambda+3 \omega_{d S} \mathrm{e}^{-\frac{3}{2} \omega_{d S} t} \sqrt{\frac{2 m}{r^{3}}}, \\
G & =G\left(a_{d S}\right)=\Lambda .
\end{aligned}
$$

The dynamical particle becomes a black hole at the time

$t_{c r}=\frac{1}{3 \omega_{d S}} \ln \left(\frac{27}{2} m \omega_{d S}\right)$,

when the pair of horizons is born. For $t>t_{c r}$ these horizons evolve as in the right panel of Fig. 1 where the dynamical cosmological horizon is approaching asymptotically to the static de Sitter event horizon.

The conclusion is that the horizons of our dynamical particles have similar evolutions as those of the McVittie $[8,12]$ or LTB space-times $[13,14]$. A minor difference is that the black hole horizons of these space-times are shrinking to the Schwarzschild sphere of radius $2 m$ while in our case the asymptotic sphere of radius $2 M(t)$ is also shrinking as $a(t)^{-3}$ and collapsing when $t \rightarrow \infty$.

\section{Radial null geodesics}

The light emitted or lensed by our dynamical particle may help us to understand if this object behaves similarly or even different from other cosmic objects studied so far. For this reason we focus now on the null geodesics in the space-times carrying these particles paying a special attention to the most interesting cases of the radial and circular trajectories.

The radial null geodesics have asymptotic forms that can be studied easily in the conformal frames $(\eta, \hat{r}, \theta, \phi)$ of the space-times $(M, a)$ where the conformal time is defined as,

$\eta=\int \frac{\mathrm{d} t}{a(t)} \rightarrow a(\eta)=a[t(\eta)]$,

and the line elements $\mathrm{d} s^{2}=a(\eta)^{2}\left(\mathrm{~d} \eta^{2}-\mathrm{d} \hat{r}^{2}\right)$ give the radial outgoing $(+)$ or ingoing $(-)$ null geodesics

$\hat{r}(\eta)=\hat{r}_{0} \pm\left(\eta-\eta_{0}\right), \quad \hat{r}_{0}=\hat{r}\left(\eta_{0}\right)$.

When a photon is emitted at the time $t$ near $\hat{r} \sim 0$ then an observed situated at the co-moving distance $\hat{d}$ can detect this photon at the instant $t^{\prime}$ which solves the outgoing equation

$\eta\left(t^{\prime}\right)=\eta(t)+\hat{d}$.

One obtains thus the scale factor $a\left(t^{\prime}\right)$ of the Lemaitre equation [20,21] of Hubble's law [22],

$1+z=\frac{a\left(t^{\prime}\right)}{a(t)}$,

which exploits the information brought by the redshift $z$ [23].

For applying the same method in the space-times $(M, a, m)$ we need to find first the equations of the radial null geodesics. This can be done in the proper frame $\{\eta, \hat{r}, \theta, \phi\}$ of the dynamical particle where the line element (31) becomes

$$
\begin{aligned}
\mathrm{d} s^{2}= & a(\eta)^{2} \\
& \times\left[\mathrm{d} \eta^{2}-\left(\mathrm{d} \hat{r}-\sqrt{\frac{2 m}{\hat{r}}} \frac{\mathrm{d} \eta}{a(\eta)^{2}}\right)^{2}+\hat{r}^{2} \mathrm{~d} \Omega^{2}\right] .
\end{aligned}
$$

Hereby we obtain the equation of the radial null geodesics

$$
\frac{\mathrm{d} \hat{r}(\eta)}{\mathrm{d} \eta}= \pm 1+\sqrt{\frac{2 m}{\hat{r}(\eta)}} \frac{1}{a(\eta)^{2}},
$$

after setting $\mathrm{d} s=0$ and $\mathrm{d} \Omega=0$. For $m=0$ this just Eq. (44) but for $m \neq 0$ we face with a non linear differential equation that cannot be integrated analytically forcing us to 
consider numerical methods or perturbations. Before starting such procedures it is convenient to substitute

$\hat{r}(\eta)=\hat{r}_{0} \pm\left(\eta-\eta_{0}\right)+\chi(\eta)$,

where the function $\chi$ which satisfies

$\frac{\mathrm{d} \chi(\eta)}{\mathrm{d} \eta}=\frac{1}{a(\eta)^{2}} \sqrt{\frac{2 m}{\hat{r}_{0} \pm\left(\eta-\eta_{0}\right)+\chi(\eta)}}$,

measures the correction due to the dynamical particle.

Perturbations with respect to the quantity $\mu=\sqrt{m}$ can be initiated starting with the expansion

$\chi(\eta) \equiv \chi(\eta, \mu)=\chi_{0}+\sum_{n} \frac{1}{n !} \mu^{n} \chi^{(n)}(\eta)$,

where $\chi_{0}$ is an arbitrary integration constant that can be adsorbed by the initial condition. We obtain thus the following algorithm

$$
\begin{aligned}
& \frac{\mathrm{d} \chi^{(n)}(\eta)}{\mathrm{d} \eta} \\
& =\left.\frac{d^{n}}{\mathrm{~d} \mu^{n}}\left[\frac{1}{a(\eta)^{2}} \frac{\sqrt{2} \mu}{\sqrt{\hat{r}_{0} \pm\left(\eta-\eta_{0}\right)+\chi(\eta, \mu)}}\right]\right|_{\mu=0} .
\end{aligned}
$$

For example, in the first order,

$$
\begin{aligned}
\chi(\eta) \simeq & \chi_{0}+\sqrt{m} \chi^{(1)}=\chi_{0} \\
& +\int_{\eta_{0}}^{\eta} \frac{\mathrm{d} \eta^{\prime}}{a\left(\eta^{\prime}\right)^{2}} \sqrt{\frac{2 m}{\hat{r}_{0} \pm\left(\eta^{\prime}-\eta_{0}\right)+\chi_{0}}},
\end{aligned}
$$

the integral can be solved in any particular case.

For testing if the corrections remain small, in the sense that these have a convenient decay in time, we focus on the first order corrections with $\chi_{0}=0$ derived for the dynamical particles discussed in our aforementioned examples. We consider first the space-time $\left(M, t^{\frac{2}{3}}, m\right)$ where the radial null geodesics in the FLRW frame, $\{t, \hat{r}, \theta, \phi\}$, can be written in the first order by solving the integral (53) as

$$
\begin{aligned}
\hat{r}(t) \simeq \hat{r}_{0} \pm 3\left(t^{\frac{1}{3}}-t_{0}^{\frac{1}{3}}\right) \\
+\sqrt{2 m}\left\{A(t) \sqrt{\hat{r}_{0} \pm 3\left(t^{\frac{1}{3}}-t_{0}^{\frac{1}{3}}\right)}-A\left(t_{0}\right) \sqrt{\hat{r}_{0}}\right. \\
+\frac{405}{8 \kappa^{\frac{7}{2}}}\left[\operatorname{Arctan} \sqrt{\frac{1}{\kappa}\left[\hat{r}_{0} \pm 3\left(t^{\frac{1}{3}}-t_{0}^{\frac{1}{3}}\right)\right]}\right. \\
\left.\left.\quad-\operatorname{Arctan} \sqrt{\frac{\hat{r}_{0}}{\kappa}}\right]\right\}
\end{aligned}
$$

where $\kappa=3 t_{0}^{\frac{1}{3}} \mp \hat{r}_{0}$ and

$A(t)=\frac{1}{\kappa t}+\frac{15}{4} \frac{1}{\kappa^{2} t^{\frac{2}{3}}}+\frac{135}{8} \frac{1}{\kappa^{3} t^{\frac{1}{3}}}$.
This solution holds for $\kappa>0$, i. e. for any ingoing null geodesic but only for the outgoing ones with $3 t_{0}^{\frac{1}{3}}>\hat{r}_{0}$. The function $A(t)$ is decreasing monotonously in time such that the first order correction remains small for any $t>t_{0}$ behaving as $t^{-\frac{1}{6}}$. Similarly, we derive the first order corrections of the radial null geodesics in the space-time $\left\{M, a_{d S}, m\right\}$ that read

$$
\begin{aligned}
\hat{r}(t) \simeq & \hat{r}_{0} \pm \frac{1}{\omega_{d S}}\left(\mathrm{e}^{-\omega_{d S} t_{0}}-\mathrm{e}^{-\omega_{d S} t}\right)+\sqrt{2 m} \\
\times & {\left[B(t) \sqrt{\hat{r}_{0} \pm \frac{1}{\omega_{d S}}\left(\mathrm{e}^{-\omega_{d S} t_{0}}-\mathrm{e}^{-\omega_{d S} t}\right)}\right.} \\
& \left.-B\left(t_{0}\right) \sqrt{\hat{r}_{0}}\right]
\end{aligned}
$$

where

$B(t)=\frac{16}{15} v^{2}+\frac{8}{15} v \mathrm{e}^{-\omega_{d S} t}+\frac{2}{5} \mathrm{e}^{-2 \omega_{d S} t}$,

with the notation $v=\omega_{d} \hat{r}_{0}+\mathrm{e}^{-\omega_{d S} t_{0}}$. We observe again that the function $B(t)$ has a time decay and the first order correction evolves conveniently as $\mathrm{e}^{-\frac{1}{2} \omega_{d S} t}$.

The next step might be the derivation of the propagation time of a photon emitted by a source near $r \sim 0$ and detected then by a remote observer, as in the case of the FLRW spacetimes. Unfortunately, this is impossible because of the technical difficulties in solving non-algebraic equations of higher orders. Therefore, numerical method are needed for finding how the presence of the dynamical particles corrects the Lemaittre equation helping us to improve the interpretation of the observational data trying to fix the actual tensions [24].

\section{Circular null geodesics}

The circular null geodesics around black holes determine the photon spheres and the angular dimensions of the shadows measured by remote observers. Turning back to the physical proper frames of the dynamical particles in the spacetimes $(M, a, m)$ with line elements (25), we consider the null geodesics, $x=x(\lambda)$, in the equatorial plane (with $\theta=\frac{\pi}{2}$ ) depending on the affine parameter $\lambda$ (which obeys $\mathrm{d} s=m_{\text {test }} \mathrm{d} \lambda$ for the massive test particles). The orthogonal component of the angular momentum, $L_{3}=L=r^{2} \frac{\mathrm{d} \phi}{\mathrm{d} \lambda}$, is conserved allowing us to write the four-velocity along the null geodesic as

$U^{0}=\frac{\mathrm{d} t}{\mathrm{~d} \lambda}, \quad U^{r}=\frac{\mathrm{d} r}{\mathrm{~d} \phi} \frac{\mathrm{d} \phi}{\mathrm{d} \lambda}=\frac{\mathrm{d} r}{\mathrm{~d} \phi} \frac{L}{r^{2}}$,

$U^{\theta}=0, \quad U^{\phi}=\frac{\mathrm{d} \phi}{\mathrm{d} \lambda}=\frac{L}{r^{2}}$. 
The line element (25) gives the prime integral

$$
\left(\frac{\mathrm{d} r}{\mathrm{~d} \phi}\right)^{2}-\frac{E^{2}}{L^{2}} r^{4}+r^{2}\left[1-h(t, r)^{2}\right]=0,
$$

where $h(t, r)$ is the function (26). Here we denoted by

$E=g_{0 \mu} U^{\mu}=\left[1-h(t, r)^{2}\right] U^{0}+h(t, r) U^{r}$,

the photon energy along the null geodesic which now is no longer conserved such that, according to the geodesic equations of $U^{t}$ and $U^{r}$, we may deduce

$\frac{\mathrm{d} E}{\mathrm{~d} \lambda}=U^{0}\left[U^{r}-h(t, r) U^{0}\right] \partial_{t} h(t, r)$.

Equations (59)-(61) are now enough for solving the problem of the null geodesics but, unfortunately, this system is complicated because of the function $h(t, r)$ depending on both the variables $t$ and $r$.

For a rapid inspection of the distinctive features of these geodesics we use here an approximation providing us with some tentative results without resorting to much more laborious methods exceeding this work. We assume that in a time domain where the system evolves very slowly this can be frozen at a given time $t$ when we can approximate

$a(t) \sim 1, \quad \frac{\dot{a}(t)}{a(t)} \sim \omega \ll 1 \rightarrow \partial_{t} h(t, r) \sim 0$,

and the energy behaves closely to a constant, $E \simeq$ const. An example could be the space-time $\left(M, a_{d S}, m\right)$ with a very small Hubble-de Sitter constant around $t \sim 0$. For finding the circular geodesics we derive first Eq. (59) with respect to $\phi$ imposing the condition $\frac{\mathrm{d}^{2} r}{\mathrm{~d} \phi^{2}}=0$ for any $\frac{\mathrm{d} r}{\mathrm{~d} \phi} \neq 0$. Hereby we obtain the constant

$\frac{E^{2}}{L^{2}}=\frac{1}{4 r^{3}}\left(2 r-2 m-4 \omega^{2} r^{3}-5 \sqrt{2 m} \omega r^{\frac{3}{2}}\right)$,

which has to be substituted in Eq. (59) where we take now $\frac{\mathrm{d} r}{\mathrm{~d} \phi}=0$ finding the new independent equation

$3 \sqrt{2 m} \omega r^{\frac{3}{2}}+6 m-2 r=0$.

Finally, we restore the time dependence substituting in both the above equations

$\omega \rightarrow r_{a}(t)^{-1}, \quad m \rightarrow M(t), \quad r \rightarrow r_{p h}(t)$,

where $r_{p h}(t)$ denotes now the dynamical radius of the photon sphere. Furthermore, we solve Eq. (64) as in the Appendix obtaining the only solution with physical meaning (A.7) that hold for $t>t_{p h}$ where $t_{p h}$ is the solution of the equation $\Delta\left(t_{p h}\right)=0$. Therefore, the photon sphere of radius

$r_{p h}(t)=3 M(t)\left[1+9 \sqrt{\frac{3}{2}} \frac{M(t)}{r_{a}(t)}+\mathscr{O}\left(\frac{M(t)^{2}}{r_{a}(t)^{2}}\right)\right]$,

appears at the instant $t_{p h}$, shrinking then to the sphere of radius $3 M(t)$. In Fig. 2 we see that in expanding universes
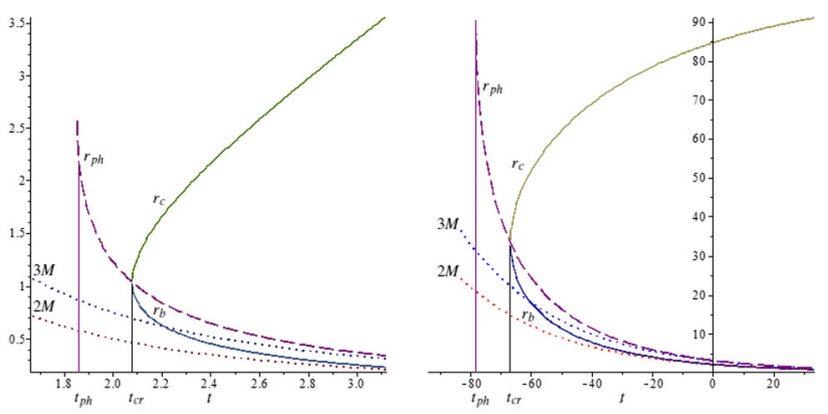

Fig. 2 The radii of the photon spheres (dashed lines) of the dynamical particles in the matter-dominated universe (left panel) and de Sitter expanding universe (right panel). The photon spheres are formed before the event horizons which appear at the time $t_{c r}$ just on the photon spheres

$t_{p h}<t_{c r}$ which means that the dynamical particle gets a photon sphere before becoming a black hole. It is remarkable that the horizons are generated simultaneously just on the photon sphere at the critical time $t_{c r}$ when $r_{p h}\left(t_{c r}\right)=$ $r_{b}\left(t_{c r}\right)=r_{c}\left(t_{c r}\right)$.

The photons can escape from the photon sphere along the spiral geodesics which are rolled out on this sphere. From the point of view of a remote observer $O$, situated in the asymptotic zone, the photon trajectory is a null geodesic in the space-time $(M, a)$ coming from an apparent source $S$. We assume that at the initial time $t$, when the distance $S O$ is $d(t)=\hat{d} a(t)$, a photon is emitted with the conserved momentum $\mathbf{k}$, energy $k^{0}=|\mathbf{k}|=E(t)$ and conserved angular momentum $L$. The apparent position of the source $S$ is on a sphere surrounding the dynamical particle whose radius at the initial time, $r_{S}(t)$, satisfies $L=r_{S}(t)|\mathbf{k}|=r_{S}(t) E(t)$. Therefore, after substituting the satisfactory approximation $r_{p h}(t) \simeq 3 M(t)$ in Eq. (63), we obtain the source radius

$r_{S}(t)=\frac{L}{E(t)} \simeq \frac{3 \sqrt{3} M(t)}{\sqrt{1-\frac{15 \sqrt{6}}{4} \frac{M(t)}{r_{a}(t)}-27 \frac{M(t)^{2}}{r_{a}(t)^{2}}}}$,

giving the angular radius [25]

$\sin \alpha(t)=\frac{r_{S}(t)}{d(t)} \stackrel{r_{a}(t) \gg M(t)}{\longrightarrow} \frac{3 \sqrt{3} M(t)}{d(t)}$,

of the shadow produced by the dynamical particle. This can be measured thanks to the conservation of the photon momentum in the spatially flat space-times $(M, a)$. The photon is detected in $O$ as having the same momentum $\mathbf{k}$ at the final time $t^{\prime}>t$ related to the redshift according to Eq. (46).

These preliminary results indicate that something new may be here in the dynamics of the horizons and photon spheres reflected in the analytical results. For example, the last term of the denominator of the radius (67) is known from the case of the Schwarzschild-de Sitter black hole [26] but the second term, which is dominant, is due to the new coupling to gravity producing our dynamical particles. Obviously, for 
$r_{a}(t) \gg M(t)$ this term as well as the last one tend to zero and we recover the well-known Synge's result [27] but depending on dynamical quantities as in Eq. (68).

\section{Concluding remarks}

We studied a family of space-times of dynamical particles produced by central singularities of densities without affecting the pressures of the asymptotic perfect fluids. For $t<t_{p h}$ the dynamical particle is a naked singularity without distinctive features but at the instant $t_{p h}$ this gets a photon sphere and implicitly a shadow creating a black hole illusion for a remote observer. The dynamical particle becomes a black hole at the instant $t_{c r}>t_{p h}$ when the pair of horizons appears on the photon sphere. For $t>t_{c r}$ the horizons evolve creating a "physical" zone between their spheres which traps the photons that evolve behind the cosmological horizon. Therefore, at the instant when a remote observer detects the photon this is already inside the cosmological horizon. This suggests that it is less probable the remote observer should record the transfiguration of our dynamical particle from a naked singularity to a black hole.

The behaviour of these particles suggests us that the family of metrics studied here could be new. The principal argument is that, in contrast with the McVittie dynamical particles, our particle produces a central singularity of the density instead of the pressure of the perfect fluid. However, technically speaking the metric (33) is close to the LTB ones apart from the time dependence which is of the FLRW type. Therefore, for testing if this difference is enough for leading to different physical results, it is useful to discuss the example of our dynamical particle embedded in the matter-dominated universe with the scale factor $a(t)=t^{\frac{2}{3}}$ and density (38). The similar solution in the LTB approach predicts a scale factor of the form $a(t)=(1+t \text { const. })^{\frac{2}{3}}$ and a density $\rho=$ const $^{\prime} . t^{-2}$ which is independent on $r$ [13]. As our density carries the singularity in $r=0$ we may conclude that these two solutions are different. Moreover, the above scale factors are translated each other, fixing different instants of big bang, in a FLRW space-time which is not invariant under time translations. These arguments justify our hope the metrics presented here are new and deserve to be studied carefully.

Data Availability Statement This manuscript has no associated data or the data will not be deposited. [Authors' comment: This theoretical work does not use or produce numerical data.]

Open Access This article is licensed under a Creative Commons Attribution 4.0 International License, which permits use, sharing, adaptation, distribution and reproduction in any medium or format, as long as you give appropriate credit to the original author(s) and the source, provide a link to the Creative Commons licence, and indicate if changes were made. The images or other third party material in this article are included in the article's Creative Commons licence, unless indi- cated otherwise in a credit line to the material. If material is not included in the article's Creative Commons licence and your intended use is not permitted by statutory regulation or exceeds the permitted use, you will need to obtain permission directly from the copyright holder. To view a copy of this licence, visit http://creativecomm ons.org/licenses/by/4.0/.

Funded by $\mathrm{SCOAP}^{3}$.

\section{Appendix A: Solving cubic equations}

Transforming the function $h(t, r) \rightarrow h\left(t, x^{2}\right)$ we obtain the cubic depressed equation $h\left(t, x^{2}\right)=1$ that reads

$x^{3}-r_{a}(t) x+r_{a}(t) \sqrt{2 M(t)}=0$,

in terms of the dynamical mass (27) and the radius (11) of the asymptotic apparent horizon. The real positive solutions we are looking for arise for $t>t_{c r}$ where the critical time $t_{c r}$ is the instant when the discriminant $\Delta(t)=$ $2 r_{a}(t)^{2}\left[2 r_{a}(t)-27 M(t)\right]$ vanishes, $\Delta\left(t_{c r}\right)=0$. We find the solutions $x_{1}, x_{2}>0$ giving the horizon radii

$r_{c}(t)=x_{1}^{2}=\frac{4}{3} r_{a}(t)\{\Re \mathscr{A}(t)\}^{2}$,

$r_{b}(t)=x_{2}^{2}=\frac{4}{3} r_{a}(t)\left\{\Re\left[\mathscr{A}(t) \mathrm{e}^{i \frac{\pi}{3}}\right]\right\}^{2}$,

where

$$
\begin{aligned}
\mathscr{A}(t)= & \left(\frac{1}{6 r_{a}(t)}\right)^{\frac{1}{6}} \\
& \times\left[i \sqrt{3} \sqrt{2 r_{a}(t)-27 M(t)}-9 \sqrt{M(t)}\right]^{\frac{1}{3}} .
\end{aligned}
$$

Equation (64) can be transformed in a cubic depressed equation,

$y^{3}-\frac{2}{27} \frac{r_{a}(t)^{2}}{M(t)} y+r_{a}(t) \sqrt{2 M(t)}-\frac{4 \sqrt{2}}{729}\left(\frac{r_{a}(t)}{\sqrt{M(t)}}\right)^{3}=0$,

after substituting $\omega \rightarrow r_{a}(t)^{-1}, m \rightarrow M(t)$ and

$\sqrt{r} \rightarrow y+y_{0}, \quad y_{0}=\frac{\sqrt{2}}{9} \frac{r_{a}(t)}{\sqrt{M(t)}}$.

When $\underline{\Delta}(t)=2 \sqrt{2} r_{a}(t)-27 M(t)>0$ we find only one real valued solution with physical meaning, $y$, giving the radius of the photon sphere

$r_{p h}(t)=\left(y+y_{0}\right)^{2}=\frac{2}{81} \frac{r_{a}(t)^{2}}{M(t)}\left\{2 \Re\left[\mathscr{B}(t) \mathrm{e}^{-i \frac{\pi}{3}}\right]+1\right\}^{2}$,

where

$\mathscr{B}(t)=\left[-i \frac{27}{4} \frac{M(t)}{r_{a}(t)} \sqrt{8-729 \frac{M(t)^{2}}{r_{a}(t)^{2}}}\right.$ 


$$
\left.+\frac{729}{4} \frac{M(t)^{2}}{r_{a}(t)^{2}}-1\right]^{\frac{1}{3}} .
$$

This solution arises at the time $t_{p h}$ which solves the equation $\Delta\left(t_{p h}\right)=0$. In expanding space-times we have $t_{p h}<t_{c r}$.

\section{References}

1. V.P. Frolov, A. Zelnikov, Introduction to Black Hole Physics (Oxford Univ. Press. Inc., New York, 2011)

2. H. Bondi, Nature 186, 535 (1960)

3. H. Bondi, M.G.J. van der Burg, A.W.K. Metzner, Proc. R. Soc. Lond. A 269, 21 (1962)

4. R. Sachs, Proc. R. Soc. Lond. A 270, 103 (1962)

5. G.C. McVittie, MNRAS 93, 325 (1933)

6. P. Painleve, C.R. Acad, Science (Paris) 173, 677 (1921)

7. A. Gullstrand, Arkiv. Mat. Astron. Fys. 16, 1 (1922)

8. B.C. Nolan, Phys. Rev. D 58, 064006 (1998)

9. M. Carrera, D. Giulini, Phys. Rev. D 81, 043521 (2010)

10. M. Carrera, D. Giulini, Rev. Mod. Phys. 82, 169 (2010)

11. R. Nandra, A.N. Lasenby, M.P. Hobson, MNRAS 422, 2931 (2012)

12. V. Faraoni, A.F.Z. Moreno, R. Nandra, Phys. Rev. D 85, 083526 (2012)
13. C. Gao, X. Chen, Y.-G. Shen, V. Faraoni, Phys. Rev. D 84, 104047 (2011)

14. I. Booth, L. Brits, J.A. Gonzalez, V. Van den Broeck, Class. Quantum Gravity 23, 413 (2006)

15. H. Bondi, Mon. Not. R. Astron. Soc. 107, 410 (1947)

16. A. Ashtekar, G.J. Galloway, Adv. Theor. Math. Phys. 9, 1 (2005)

17. A.B. Nielsen, M. Visser, Class. Quantum Gravity 23, 4637 (2006)

18. W. Rindler, MNRAS 116, 662 (1956)

19. W. Rindler, Essential of Relativity (Springer, Heidelberg, 1969)

20. G.E. Lemaître, Ann. Soc. Sci. de Bruxelles 47A, 49 (1927)

21. G.E. Lemaître, MNRAS 91, 483 (1931)

22. E. Hubble, Proc. Natl. Acad. Sci. 15, 168 (1929)

23. E. Harrison, Astrophys. J. 403, 28 (1993)

24. L. Verde, T. Treu, A. Riess, Nat. Astron. 3, 891 (2019)

25. I.I. Cotăescu, Eur. Phys. J. C 81, 32 (2021)

26. V. Perlick, OYu. Tsupko, G.S. Bisnovatyi-Kogan, Phys. Rev. D 97, $104062(2018)$

27. J.L. Synge, Mon. Not. R. Astron. Soc. 131, 463 (1966) 